\section{ORIGINAL RESEARCH}

\author{
M.L. Escolar \\ M.D. Poe \\ J.K. Smith \\ J.H. Gilmore \\ J. Kurtzberg \\ W. Lin \\ M. Styner
}

\title{
Diffusion Tensor Imaging Detects Abnormalities in the Corticospinal Tracts of Neonates with Infantile Krabbe Disease
}

\begin{abstract}
BACKGROUND AND PURPOSE: It is not possible to determine if neonates diagnosed with Krabbe disease through statewide neonate screening programs will develop the disease as infants, juveniles, or adults. The only available treatment for this fatal neurodegenerative condition is unrelated umbilical cord transplantation, but this treatment is only effective before clinical symptoms appear. Therefore, a marker of disease progression is needed. The purpose of this study was to evaluate the use of diffusion tensor imaging (DTI) with fiber tracking in identifying early changes in major motor tracts of asymptomatic neonates with infantile Krabbe disease.
\end{abstract}

\begin{abstract}
MATERIALS AND METHODS: Six neonates with infantile Krabbe disease identified because of family history underwent brain MR imaging within the first 4 weeks of life. Six-direction DTI and quantitative tractography of the corticospinal tracts were performed. Hypothesis tests, 1 for each hemisphere, were used to determine whether the fractional anisotropy (FA) ratio of the neonates with infantile Krabbe disease was significantly different from that of 45 age- and sex-matched controls.
\end{abstract}

RESULTS: The average FA ratio for patients with Krabbe disease was 0.89 and 0.87 for left and right tracts, respectively $(P=.002$ and $<.001)$. After adjusting for gestational age, gestational age at birth, birth weight, sex, and race, the 6 patients with Krabbe disease had significantly lower FA values than the controls $(P<.001)$

CONCLUSIONS: DTI with quantitative tractography detected significant differences in the corticospinal tracts of asymptomatic neonates who had the early-onset form of Krabbe disease. Once standardized and validated, this tool has the potential to be used as a marker of disease progression in neonates diagnosed through statewide neonate screening programs.

$K^{\mathrm{r}}$ rabbe disease is an autosomal recessive disorder characterzed by a deficiency of the lysosomal enzyme galactocerebrosidase (GALC), which aids in the breakdown and removal of galactolipids found in myelin. The accumulation of galactolipids results in inflammation, rapid demyelination, and death within 2 years. ${ }^{1}$ This is a very rare disorder with an incidence of 1 in 70-100,000 in live births. ${ }^{1}$ The phenotypic expressions of the mutations vary widely, with the disease presenting as an infantile, juvenile, or adult form. The infantile presentation has an onset within the first 6 months of life and is the most frequent form of presentation. Umbilical cord blood transplantation is the only available treatment and is only effective before symptoms develop. ${ }^{2}$ Treated infants preserve cognitive function but develop some degree of motor disability, which ranges from mild to severe.

Received August 29, 2008; accepted after revision December 5.

From the Program for Neurodevelopmental Function in Rare Disorders at the Center for the Study of Development and Learning (M.L.E.), FPG Child Development Institute (M.D.P.), Departments of Radiology (J.K.S., W.L.), Psychiatry (J.H.G., M.S.), and Computer Science (M.S.), University of North Carolina at Chapel Hill, Chapel Hill, NC; and Pediatric Blood and Marrow Transplantation Program (J.K.), Duke University Medical Center, Durham, NC.

This work was supported by the National Institutes of Health (NIH) Roadmap for Medical Research, Grant U54 EB005149-01; the Neurodevelopmental Research Center at UNC-CH (NIH P3OHD03110); and the National Institute of Mental Health Conte Center (MH064065), which funded the image processing and control data.

Please address correspondence to Maria L. Escolar, MD, 1450 Raleigh Rd, University of North Carolina at Chapel Hill, Chapel Hill, NC 27517; e-mail: maria.escolar@cdl.unc.edu

Indicates open access to non-subscribers at www.ajnr.org

DOI 10.3174/ajnr.A1476
With the implementation of statewide mandatory neonate screening programs, neonates with no previous family history are being diagnosed. Among the neonates who screen positive for Krabbe disease, it is not possible to identify with certainty which ones will develop the infantile, juvenile, or adult forms because phenotype predictions cannot be made a priori from the genetic mutation or degree of enzyme deficiency. Therefore, the development of a predictive measure that identifies changes in the brain before symptoms develop could potentially clarify which neonates will develop the infantile form. This information is critical for clinicians counseling parents about the treatment of a neonate who screens positive for Krabbe disease. A better understanding of markers of disease onset and expected phenotype after treatment would also help parents and clinicians make decisions about the optimal time of treatment.

Mild hyperintense signal-intensity abnormalities in the internal capsule have been previously reported in untreated neonates with Krabbe disease. ${ }^{3}$ These findings coupled with reports showing rapid increase in fractional anisotropy (FA) with age $e^{4}$ and in the central regions of the internal capsule $e^{5-9}$ lead us to believe that quantifying these changes may help identify which asymptomatic babies should be treated because they are more likely to progress rapidly and die.

The purpose of this study was to evaluate whether diffusion tensor imaging (DTI) with quantitative tractography can detect early changes in major motor tracts before clinical symptoms develop. We further describe the relationship between changes in these motor tracts and degree of motor disability at 12 months of age. 


\section{Materials and Methods}

Neonates with Krabbe disease, identified by family history or through the New York State screening program, were referred to the Program for Neurodevelopmental Function in Rare Disorders at the University of North Carolina at Chapel Hill for assessment of baseline neurologic function before receiving unrelated umbilical cord blood transplantation at Duke University Medical Center. The assessment included a detailed neurodevelopmental evaluation concurrent with a brain $\mathrm{MR}$ imaging within the first 4 weeks of life. A follow-up evaluation of motor function was performed at 6, 9, 12, and 24 months. Six neonates with infantile Krabbe disease diagnosed prenatally or at birth because of family history were scanned without sedation on an Allegra 3T head-only MR scanner (Magnetom Allegra; Siemens, Erlangen, Germany). Neonatal controls were recruited as part of a separate study and were scanned on the same scanner by using an identical protocol. ${ }^{10}$ The studies were approved by the institutional review board at the University of North Carolina.

Neurodevelopmental Assessments. The Peabody Developmental Motor Scales-2, ${ }^{11}$ the Mullen Scales of Early Learning, ${ }^{12}$ and the Protocol for the Assessment of Neurodevelopment in Infants (M.L.E.; unpublished data) were used to assess cognitive and motor development in children with Krabbe disease. Age equivalents were used to track development with time and compare across tests.

Imaging. The details of the image acquisition and processing have been reported in a previous publication. ${ }^{10}$ The 3 main imaging sequences were a magnetization-prepared rapid acquisition of gradient echo T1-weighted, a proton-density/T2 turbo spin-echo, and a single-shot echo-planar DTI. The imaging parameters for the DTI sequence were as follows: $\mathrm{TR}=4219 \mathrm{~ms}$; TE $=92.2 \mathrm{~ms}$; in-plane resolution $=2 \times 2 \mathrm{~mm}^{2}$ with a section thickness of $2 \mathrm{~mm}, 6$ diffusion directions, and 10 averages. Seven images were acquired, 1 without a diffusion gradient $(b=0)$ and the remaining 6 with $b=1000 \mathrm{~s} / \mathrm{mm}^{2}$. Before the imaging session, neonates were fed, swaddled, and fitted with ear protection and had their heads fixed in a Vac-Fix device (Vak-Lok; MedTec, Orange City, Iowa). A pulse oximeter was used to monitor heart rate and oxygen saturation.

DTI Processing. The processing protocol consisted of multiple repeated DTI acquisitions, which were coregistered, filtered for bad sections, and finally averaged via an automatic DTI quality control tool, called DTIChecker (http://www.ia.unc.edu/dev/download/ dtichecker). The final DTI dataset was fed into a fiber tracking tool, which automatically computes the FA and mean diffusivity (MD) maps. Regions of interest were outlined on the base $(b=0)$ image for regional FA and MD analysis and for DTI fiber tracking by using our ITK-SNAP (http://www.itksnap.org) ${ }^{13}$ tool. For the analysis plan, the fiber circuitries of interest were determined in collaboration with a neuroanatomist (James Fallon, University of California, Irvine, Calif). Our fiber tracking tool computes DTI fibers via a streamline algorithm that follows the major diffusion direction from specified source to target regions. ${ }^{14}$ DTI fibers were postprocessed by using our inhouse Fiber Viewer tool by removing outliers grouped into separate left and right hemispheric tracts. Correspondence along all fibers was established via a user-selected origin for each fiber bundle and an equal step parameterization along every fiber. On the basis of this correspondence, the mean and SD of FA and MD across fiber bundles were plotted in curves and analyzed along the DTI fiber bundles. ${ }^{15}$

After the fiber tracts were defined, mean FA values were calculated at twenty 3-mm intervals along the tracts. However, because preliminary data have shown that the central and peripheral regions of the tracts show increased variability due to a low signal intensity-to-noise

\begin{tabular}{|c|c|c|c|c|}
\hline \multirow[b]{2}{*}{ Descriptive Information } & \multicolumn{2}{|c|}{$\begin{array}{l}\text { Krabbe } \\
(n=6)\end{array}$} & \multicolumn{2}{|c|}{$\begin{array}{c}\text { Controls } \\
(n=45)\end{array}$} \\
\hline & Mean & SD & Mean & SD \\
\hline Birth weight $(\mathrm{g})$ & 3.13 & 0.65 & 3.45 & 0.45 \\
\hline Gestational age birth (wk) & 38.3 & 1.5 & 39.7 & 1.0 \\
\hline Gestational age at scanning (wk) & 40.1 & 1.9 & 42.9 & 1.7 \\
\hline Male & $50 \%$ & & $60 \%$ & \\
\hline White & $100 \%$ & & $84 \%$ & \\
\hline
\end{tabular}

ratio, only the $30-\mathrm{mm}$ segment near the internal capsule was used to index FA and MD. Previous studies have shown comparisons of specific distances along the tracts (eg, at 30 or $55 \mathrm{~mm}$ ) to be highly sensitive to the spatial alignment of the tracts between subjects ${ }^{16}$; by taking the mean of this segment, we increased both the precision of the measure and the power to detect differences.

\section{Statistical Methods}

The FA value for patients with Krabbe disease was scaled in terms of the expected FA in typical neonates. To calculate this ratio, we estimated an expected mean value based on the age at scanning, gestational age at birth, sex, and birth weight on the basis of a general linear model that was fitted to the 45 typical neonates. The outcome variable was the mean FA value for the $30-\mathrm{mm}$ segment with age at scanning, gestational age, sex, and birth weight as predictors. The left and right tracks were modeled separately. The coefficients from these 2 models were then used to calculate the expected mean FA values for the left and right tracks of each of the 6 patients with Krabbe disease. The actual mean FA values of the patients with Krabbe disease were then divided by the expected value based on the predictors, producing a ratio that represents the percentage of normal FA.

To test for mean and slope differences between the patients with Krabbe disease and typical controls, we fitted a mixed regression model with repeated measures between the 2 hemispheres (left/right). The model regressed group, corrected age at scanning, hemisphere (left/right), the interaction terms between group and left and group and hemisphere, on FA ratio. The difference between the FA ratio of the Krabbe babies and the typical controls was tested for significance at 40 weeks corrected age at scan.

\section{Results}

Four neonates with Krabbe disease identified by family history and 2 identified through neonate screening were evaluated between December 2004 and September 2007. Their corrected age at the time of the MR imaging ranged from 1 to 3 weeks (38-42 weeks from gestation) and birth weight ranged from 2.28 to $4.13 \mathrm{~kg}$. There were 3 females and 3 males (Table 1). All patients were subsequently transplanted with unrelated umbilical cord blood transplantation. Two patients died of transplant-related complications, 1 of vaso-occlusive disease and 1 of persistent pulmonary hypertension. The 4 patients who survived now range in age from 18 months to 4 years. The patients with Krabbe disease generally weighed less at birth and were younger than the controls at both birth and at the time of the scanning. However, these variables were controlled for in all the statistical tests. Table 1 shows descriptive information for the 6 patients with Krabbe disease and the 45 controls. Of the 6 patients, 5 had relatively normal neurologic examination findings and were classified as stage $1 ; 1$ patient had minimal 


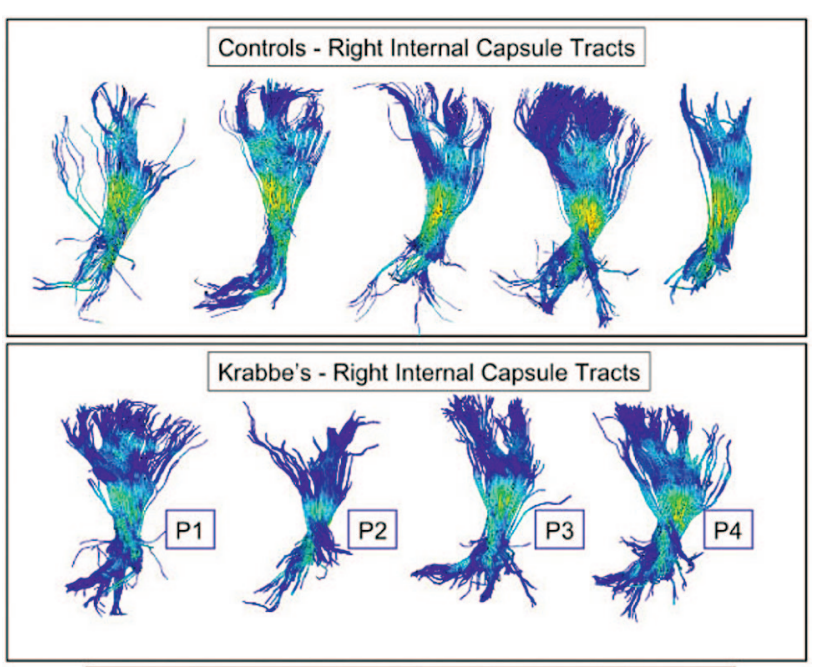

$\mathrm{FA}=0 \quad \mathrm{FA}=1$

Fig 1. Visualization of DTI fiber tracts through the right hemispheric internal capsule with FA coloring of the fibers from a lateral viewpoint. Top row: 5 representative control subjects. Bottom row: 4 selected Krabbe subjects. Visually, all Krabbe subjects show lower FA values along most of the fiber bundle than any of the controls.

neurologic symptoms, clonus in 1 lower extremity, and was classified as stage $2 .^{2}$

When the fiber tracts were visualized by color coding for FA values in both controls and patients with Krabbe disease, the normal pattern of myelination that goes from the center to the periphery was evident in both groups. However, the Krabbe group showed lower FA values along most of the fiber bundle than any of the controls and lower FA values toward the periphery (Fig 1). These images produced by our Fiber Viewer software are indicative of a more immature pattern of myelination in the Krabbe group.

The mean FA of the central area of the corticospinal tracts in the controls showed a clear trend of increasing FA with age (Fig 2). However, this trend was less clear in the patients with Krabbe disease. After adjusting for gestational age, gestational age at birth, birth weight, sex, and race, the 6 patients with Krabbe disease had significantly lower FA values than the controls $(P<.001)$. Figure 3 represents the FA ratios for the 6 patients with Krabbe disease and the 45 controls. The average FA ratios for the patients with Krabbe disease were 0.89 and 0.87 for left and right tracts, respectively $(P=.002$ and $P<$ $.001)$. Although it appears that the FA values become more abnormal with increasing age, this effect is not statistically significant $(P=.12)$. There was no significant difference between the FA values of the left and right hemispheres $(P=.58)$ or in the mean FA by group and location (right/left) (Table 2).

Because 2 of the children with Krabbe fell outside the range of our controls, we reanalyzed the data excluding these 2 children from the analysis. Using only the children who were $\geq 40$ weeks at the time of scanning, we found that the differences between the Krabbe group and the controls increased to 0.18 and $0.20(P=.002$ and $P<.001)$ for left and right hemispheres.

All 4 children are within the range of age-appropriate development in cognitive, receptive language, and fine motor skills. However, 3 of the 4 patients had gross motor delay at the last follow-up visit. The neurodevelopmental trajectories in the motor area were correlated to the mean FA ratios in the 4 surviving patients. Of the 2 patients with the highest FA, one can walk and run at 4 years of age and the other can walk with a walker at 36 months. The patient with the lowest neonatal FA ratio shows the greatest delay in the motor area, not being able to walk independently at 25 months of age. Another patient with low FA is not able to walk at 18 months and has the development of a 13-month-old. All patients are cognitively healthy.

\section{Discussion}

We have demonstrated in a very small sample of patients with Krabbe disease that DTI with a quantitative tractography methodology can detect significant differences in the corticospinal tracts of neonates with Krabbe disease before clinical symptoms develop. All of the 6 patients with infantile Krabbe disease had lower FA values across the DTI fiber bundle of the internal capsule compared with healthy age-matched controls. The average FA ratio for the patients with Krabbe disease was 0.89 and 0.87 for the left and right tracts, respectively, with a significant difference $(P<.001)$ in FA values between Krabbe and control values. Most interesting, we also found a strong trend, albeit nonsignificant, in FA with age, with the Krabbe group's symptoms becoming progressively abnormal over a matter of weeks. Of the 4 children followed $>12$ months after transplantation, the children with FA values around $0.90(0.89$ and 0.91 ) are performing better in standardized assessments than the children with FA values of 0.73 and 0.84 .

A previous study has shown normal myelination and axonal integrity as measured by DTI methods in babies with Krabbe disease. ${ }^{3}$ However, their methodology was very different from ours. First, the authors looked at many regions that do not show early myelination and thus are less likely to show a difference. Second, when they looked at the internal capsule, they used the manual region-of-interest-based methodology, which often mixes different fibers that cross the same region. Our methodology nicely picks out only those image regions that are associated with the corticospinal tract.

The early abnormalities in FA seen in our study in the internal capsule, a major motor pathway, may explain the plateau in motor development seen in treated babies with Krabbe disease even in the presence of improved overall brain myelination. ${ }^{17}$ Previous studies have shown that FA appears to be dependent on tract organization. ${ }^{18}$ This disruption could also explain the motor impairment that may be present even before myelination is disrupted by the lack of GALC. Further studies in animal models looking at the influence of GALC in tract development would be needed to confirm this hypothesis.

The greatest limitation of this study is the small number of infants with Krabbe disease $(n=6)$, but this represents a very large portion of this population and the results are statistically significant. Although random sampling was not used, sequential enrollment was used in that each child who was thought to have infantile Krabbe disease who presented at our center was scanned. During the study period, our center saw nearly all presymptomatic children from the United States who were suspected of having early-infantile Krabbe disease. Therefore, there is no reason to believe that the results are not similar to those from a random sample.

Another limitation is the small number of controls who 


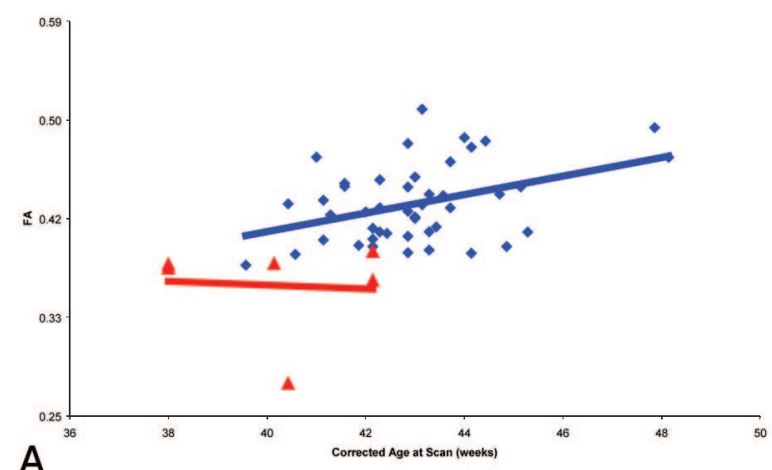

A

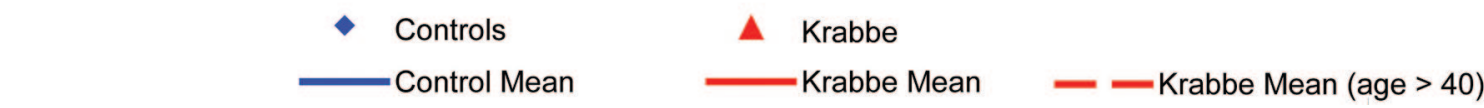

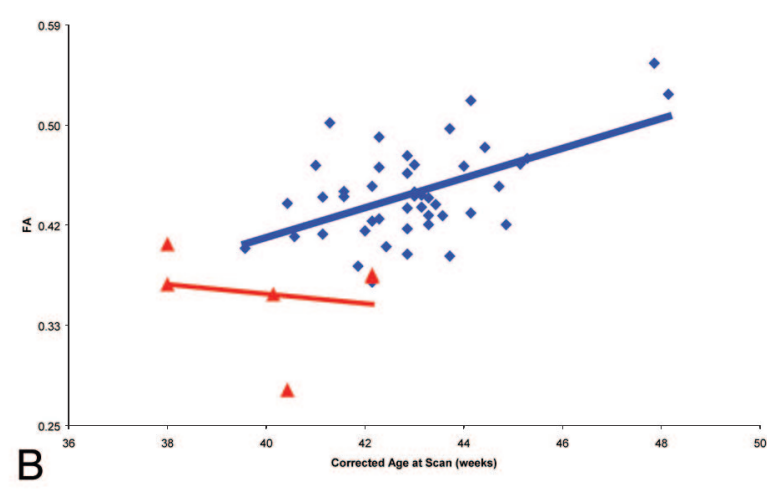

Fig 2. Raw FA mean values for patients with infantile Krabbe disease and controls. Each point represents a child's mean FA across the central portion of the corticospinal tract. The red triangles represent the 6 patients with Krabbe disease, whereas the blue diamonds show the typical controls. The red and blue lines represent the linear trend related to corrected age at the time of the MR imaging.
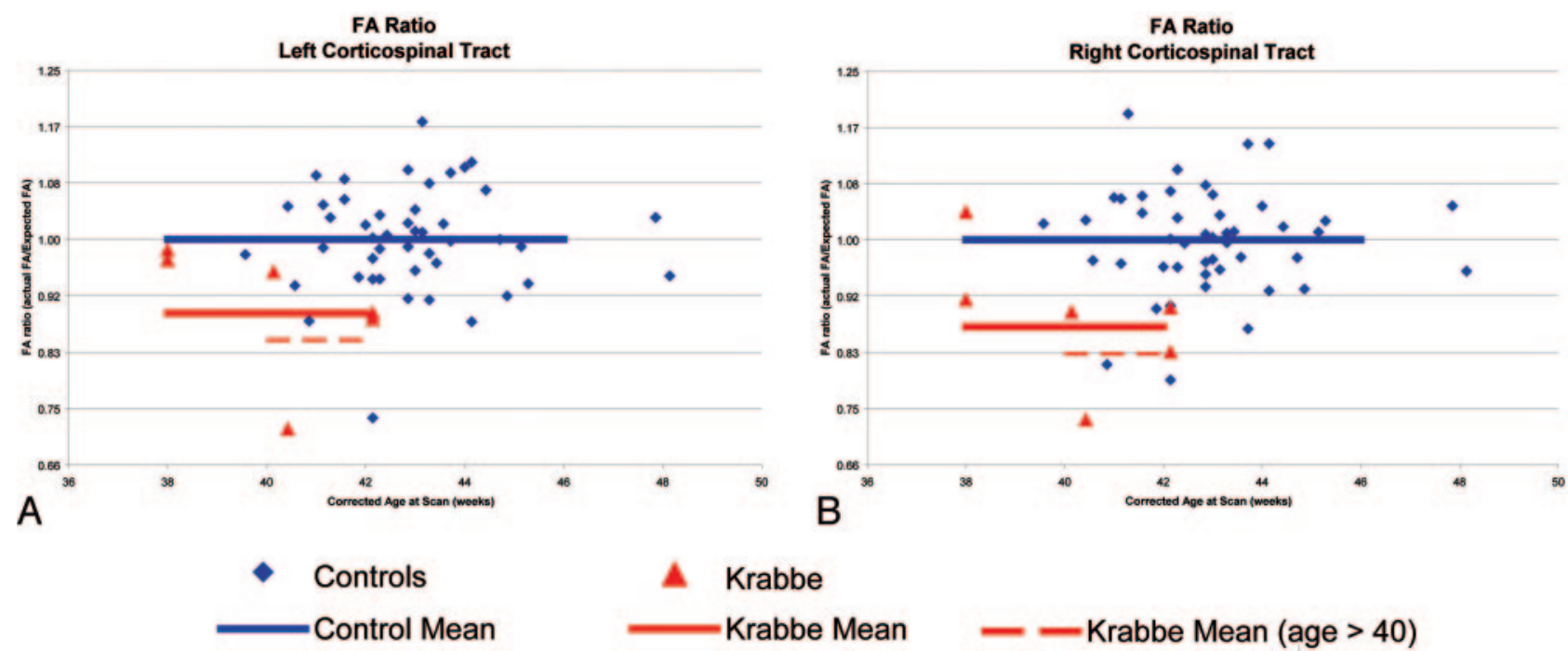

- Krabbe Mean (age > 40)

Fig 3. Normalized FA ratios for patients with infantile Krabbe disease and controls. The FA ratio is the observed FA divided by the expected FA. given the child's gestational age at birth, corrected age at scanning, birth weight, and sex. The red triangles represent the 6 patients with Krabbe disease, whereas the blue diamonds show the typical controls. The horizontal black lines represent the SDs based on the controls.

\begin{tabular}{llllll}
\hline \multicolumn{2}{l}{ Table 2: Mean FA by group and location (right/left) } \\
\hline \multicolumn{2}{c}{ Krabbe } & & \multicolumn{2}{c}{ Controls } \\
\cline { 2 - 3 } \cline { 5 - 6 } Variable & Mean & SD & & Mean & SD \\
\hline Right & & & & & \\
Mean FA & 0.36 & 0.04 & & 0.45 & 0.04 \\
FA ratio & 0.87 & 0.09 & & 1.00 & 0.08 \\
Left & & & & \\
Mean FA & 0.36 & 0.04 & & 0.43 & 0.04 \\
FA ratio & 0.89 & 0.09 & 1.00 & 0.08 \\
\hline
\end{tabular}

Note:-FA indicates fractional anisotropy.

were scanned before 41 weeks' gestational age. Although a linear model was assumed to create estimates of typical FA values during the 38- to 41-week period, it is possible that the linear relationship observed from 41 to 48 weeks does not hold for this early period of development. However, because the
2 youngest babies showed the least differences from our estimate of typical babies, we believe our results to be a conservative estimate. Conversely, any estimates of increasing abnormality with age would be highly sensitive to any misspecification of the model during this early period. For this reason, the nonsignificant age effect should be examined with caution.

The fact that all babies showed abnormalities in motor examination confirms that these babies were going to develop Krabbe disease because the pattern of neurologic involvement has been described in previous studies ${ }^{17}$ and this pattern is not seen in babies transplanted for other diseases. We have previously published our ability to predict motor outcome based on the staging system developed by our group. ${ }^{2}$ However, this is subjective, based on the experience of the examiner and, therefore, more difficult to disseminate. Correlating development with previous DTI measures is more objective, and the methodology is easier to disseminate to sites with no experience in 
evaluating these patients. However, the prediction could be challenging due to uncontrolled environmental influences. Intervention services, length of hospitalization, and medical complications may impact a child's motor development. For example, one of the patients had higher initial values of FA than the other babies but developed graft-versus-host disease, which required high doses of steroids. The increased weight and steroid-induced myopathy affected this child's motor development. Therefore, this patient may be more delayed than would be predicted by the FA at the time of transplantation.

Patients who screen positive for low GALC but have no family history may develop early-onset disease or may be healthy or clinically silent until late childhood or even adulthood. An objective measure of disease progression is necessary to decide whether an asymptomatic baby should be treated because once clinical symptoms appear, it is too late to derive benefits from treatment. As previously reported, conventional MR images and neurophysiologic tools cannot be used to predict outcome or to correlate with the disease stage because of poor standardization of these tools in the neonatal period. ${ }^{2}$ In this study, even a small sample of patients showed significant differences between healthy controls and babies diagnosed with infantile Krabbe disease. The differences in FA in the superior and middle cerebellar peduncle may help differentiate between babies who will develop the early-onset disease, those who will develop disease at a much later time, and those who will never develop disease. This approach can provide very consistent FA values and detect small regional differences and age-related changes in the first 2 years of life. For this reason, DTI with quantitative tractography may prove to be an excellent technology for studying infantile Krabbe disease and other demyelinating conditions of early childhood. A large sample of healthy controls and babies with low enzymes who do not develop early-infantile disease will need to be studied for an adequate standardization and validation of this tool. Once fully standardized, this tool has the potential to be a key component of a population screening program to help determine who should receive treatment before symptoms develop.

\section{Acknowledgments}

We thank James H. Fallon of the University of California, Irvine, through the National Alliance for Medical Image Com- puting for insightful discussion and the provision of Fig 1 and

Sampath Vetsa for processing the Krabbe images.

\section{References}

1. Wenger DA, Suzuki K, Suzuki Y. Galactosylceramide lipidosis: globoid-cell leukodystrophy (Krabbe disease). In: Scriver CR, Beaudet AL, Sly WS, et al, eds. The Metabolic and Molecular Bases of Inherited Disease. 8th ed. New York: McGraw-Hill; 2001:3669-94

2. Escolar ML, Poe MD, Martin HR, et al. A staging system for infantile Krabbe disease to predict outcome after unrelated umbilical cord blood transplantation. Pediatrics 2006;118:e879-89

3. McGraw P, Liang L, Escolar M, et al. Krabbe disease treated with hematopoietic stem cell transplantation: serial assessment of anisotropy measurements-initial experience. Radiology 2005;236:221-30

4. Hermoye L, Saint-Martin C, Cosnard G, et al. Pediatric diffusion tensor imaging: normal database and observation of the white matter maturation in early childhood. Neuroimage 2006;29:493-504

5. Zhai G, Lin W, Wilber KP, et al. Comparisons of regional white matter diffusion in healthy neonates and adults performed with a 3.0-T head-only MR imaging unit. Radiology 2003;229:673-81

6. Zhang L, Thomas KM, Davidson MC, et al. MR quantitation of volume and diffusion changes in the developing brain. AJNR Am J Neuroradiol 2005;26: $45-49$

7. Schmithorst VJ, Wilke M, Dardzinski BJ, et al. Correlation of white matter diffusivity and anisotropy with age during childhood and adolescence: a cross-sectional diffusion-tensor MR imaging study. Radiology 2002;222: 212-18

8. Mukherjee P, Miller JH, Shimony JS, et al. Normal brain maturation during childhood: developmental trends characterized with diffusion-tensor MR imaging. Radiology 2001;221:349-58

9. Mukherjee P, Miller JH, Shimony JS, et al. Diffusion-tensor MR imaging of gray and white matter development during normal human brain maturation. AJNR Am J Neuroradiol 2002;23:1445-56

10. Gilmore JH, Zhai G, Wilber K, et al. 3 Tesla magnetic resonance imaging of the brain in newborns. Psychiatry Res 2004;132:81-85

11. Folio R, Fewell R. Peabody Developmental Motor Scales and Activity Cards. Allen, Tex: DLM Teaching Resources; 1983

12. Mullen E. The Mullen Scales of Early Learning: AGS Edition. Circle Pines, Minn: American Guidance Service; 1995

13. Yushkevich PA, Piven J, Hazlett HC, et al. User-guided 3D active contour segmentation of anatomical structures: significantly improved efficiency and reliability. Neuroimage 2006;31:1116-28. Epub 2006 Mar 20

14. Fillard P, Gilmore J, Piven J, et al. Quantitative Analysis of White Matter Fiber Properties along Geodesic Paths. In: Medical Image Computing and ComputerAssisted Intervention-MICCAI 2003. Springer; 2003:16-23

15. Corouge I, Fletcher PT, Joshi S, et al. Fiber tract-oriented statistics for quantitative diffusion tensor MRI analysis. Med Image Anal 2006;10:786-98

16. Schimrigk SK, Bellenberg B, Schluter M, et al. Diffusion tensor imaging-based fractional anisotropy quantification in the corticospinal tract of patients with amyotrophic lateral sclerosis using a probabilistic mixture model. AJNR Am J Neuroradiol 2007;28:724-30

17. Escolar ML, Poe MD, Provenzale JM, et al. Transplantation of umbilical-cord blood in babies with infantile Krabbe's disease. N Engl J Med 2005;352: 2069-81

18. Gilmore JH, Lin W, Corouge I, et al. Early postnatal development of corpus callosum and corticospinal white matter assessed with quantitative tractography. AJNR Am J Neuroradiol 2007;28:1789-95 